\title{
Optimal Targeted Temperature in Targeted Temperature Management without Automated Devices Using a Temperature Feedback System: A Prospective, Multicenter Observational Study (KoCARC Registry)
}

\author{
Taeyoung Kong \\ Yonsei University College of Medicine \\ Je Sung You \\ Yonsei University College of Medicine \\ Hye Sun Lee \\ Yonsei University College of Medicine \\ Soyoung Jeon \\ Yonsei University College of Medicine \\ Yoo Seok Park \\ Yonsei University College of Medicine \\ Sung Phil Chung ( $\nabla$ emstar@yuhs.ac) \\ Yonsei University College of Medicine
}

Research

Keywords: Out-of-hospital cardiac arrest, Targeted temperature management, Regulation, Body Temperature, Prognosis

Posted Date: July 26th, 2021

DOI: https://doi.org/10.21203/rs.3.rs-725650/v1

License: () (1) This work is licensed under a Creative Commons Attribution 4.0 International License. Read Full License 


\section{Abstract}

Background:

Targeted temperature management (TTM) at $32^{\circ} \mathrm{C}-36^{\circ} \mathrm{C}$ improves patient outcomes following out-of-hospital cardiac arrest (OHCA). TTM using automated temperature management devices with feedback systems (TFDs) is recommended, but the equipment is often unavailable. This study aimed to investigate therapeutic relations between targeted temperatures and TFDs on the outcomes of OHCA patients with TTM. To the best of our knowledge, this is the first study to identify the optimal targeted temperature in TTM without TFD with regards to neurological outcomes in OHCA patients.

\section{Methods:}

This prospective multicenter study analyzed nontraumatic OHCA registry data between October 2015 and June 2020 from 62 institutions. Patients were classified into four groups based on targeted temperatures and TFD implementation: TTM at $36^{\circ} \mathrm{C}$ with TFD (36TFD), TTM at $33^{\circ} \mathrm{C}$ with TFD $(33$ TFD), TTM at $36^{\circ} \mathrm{C}$ without TFD (36NTFD), and TTM at $33^{\circ} \mathrm{C}$ without TFD (33TNFD). Clinical outcomes were neurological status at discharge.

\section{Results:}

A total of 938 patients were included in the analysis. A significant interaction was found between targeted temperatures and TFD use on neurological outcomes $(P=0.032)$. There was an independent association between the 33NTFD patients with the least favorable neurological outcomes among the four groups after adjustment for covariates. However, no significant differences were observed in neurological outcomes among the 36TFD, 33TFD, and 36NTFD groups after adjusting for covariates. Compared to 33NTFD patients, 36NTFD patients exhibited significantly higher adjusted ORs for favorable neurological status (OR: $4.28,95 \% \mathrm{Cl}: 1.50-12.16, \mathrm{P}<0.01)$.

\section{Conclusions:}

In OHCA patients receiving TTM without TFDs, the adjusted predicted probability of favorable neurological outcomes at hospital discharge was greater for TTM at $36^{\circ} \mathrm{C}$ than that at $33^{\circ} \mathrm{C}$. The 36 NTFD group displayed similar therapeutic effect as the TFD groups. This suggests that a TTM of $36^{\circ} \mathrm{C}$ rather than $33^{\circ} \mathrm{C}$ is associated with more favorable neurological outcomes if TFDs are unavailable.

\section{Background}

Despite recent advances in emergency medicine and resuscitation care, post-cardiac arrest brain injury remains a major cause of mortality and disability [1] Targeted temperature management (TTM) is employed to reduce neurological damage of patients resuscitated from cardiac arrest [2]. Following landmark clinical trials in 2002 demonstrating that TTM at $32-34^{\circ} \mathrm{C}$ for $12-24 \mathrm{~h}$ improved neurological outcomes after out-of-hospital cardiac arrest (OHCA), widespread use of therapeutic hypothermia protocols targeting $33^{\circ} \mathrm{C}$ was implemented $[3,4]$. High-quality trials reported that TTM at $33^{\circ} \mathrm{C}$ did not confer clinical benefits in post-cardiac arrest patients compared with $36^{\circ} \mathrm{C}$ [5]. Current international guidelines recommend TTM of unconscious patients who have been resuscitated from cardiac arrest at $32-36^{\circ} \mathrm{C}$ for at least 24 hours $[6,7]$.

TTM methods can be classified into two major categories according to the temperature feedback systems [8, 9]. Temperature management devices with feedback systems (TFDs) have a controlled feedback loop that continuously measures the patients' core temperature and adjusts the temperature of the cooling element accordingly [1]. However, TTM can also be performed without the use of sophisticated controlled feedback systems by using ice and/or cold packs, fans and cold blankets, and manual temperature management which requires frequent assessment by staff $[1,10]$. TTM with feedback devices provides more accurate maintenance of targeted temperatures and prevents overcooling and rebound of hyperthermia compared to TTM without feedback devices [1, 9-11]. However, TTM without temperature feedback devices (NTFD) is still employed in situations for which equipment is unavailable for various reasons [8]. A survey of TTM in post-cardiac arrest survivors reported that only $76 \%$ of intensive care units harnessed TFDs [12]. Although several studies have compared $\mathrm{TTM}$ at $33^{\circ} \mathrm{C}$ and $36^{\circ} \mathrm{C}$ with regards to the accuracy of temperature control and post-cardiac arrest outcomes [13-16], evidence for the relationship between targeted temperatures and TFDs remains scarce. We hypothesized that the therapeutic effect of using TFDs may be different according to the targeted temperature. In this study, we investigated therapeutic relationships between targeted temperatures and TFDs on the neurological outcomes of OHCA patients with TTM. Moreover, we aimed to find the optimal temperature for OHCA patients receiving TTM without TFDs.

\section{Methods}

\section{Study design and population}

This multicenter observational study used data from the Korean Cardiac Arrest Research Consortium (KoCARC) registry, which is a web-based prospective database that collects data on patients with OHCA from 62 academic emergency departments (EDs) in the Republic of Korea [17-19]. The KoCARC is a collaborative research network developed to support studies in the field of OHCA and to foster collaborations among research groups. The registry enrolled OHCA patients who were transported to participating EDs by emergency medical services (EMSs) with resuscitation attempts and with cardiac arrests of presumed cardiac origin as determined by emergency physicians. Exclusion criteria of the KoCARC registry are OHCA due to definite non-cardiac etiologies, such as trauma, drowning, burning, hanging, poisoning, and asphyxia. Patients under hospice care, patients with a terminal illness documented by medical records, patients with pre-documented "Do Not Attempt Resuscitation" records, and pregnant women were also excluded. Researchers at each hospital were trained in data extraction from hospital medical records to ensure correct data entry into the standardized web-based electronic case report form. The case report form consisted of more than 200 variables, including demographics, cardiac arrest characteristics, past medical history, laboratory tests, EMS care, therapeutic interventions, and patient outcomes. Outliers or incorrect values were filtered using a predefined data-entry system. The KoCARC quality 
management committee, comprising physicians, local research coordinators, and investigators from each participating institution, regularly monitored and reviewed data quality. The KoCARC registry collecting protocol was reviewed and approved by the institutional review board of each participating hospital. This study was purely observational, and therefore, the institutional review board waived the need for informed consent. The KoCARC registry was registered at clinicaltrials.gov as protocol NCT03222999 [18].

Patients in the KoCARC registry who received TTM from October 2015 to June 2020 were included in this study. Patients younger than 18 years of age, patients with the provision of extracorporeal CPR, patients with incomplete information about TTM and prognosis, and patients who died in the ED prior to ICU admission were excluded. Patients transferred from other hospitals were excluded if they had spent $>24$ hours in other hospitals. TTM was defined as the control of core body temperature to the targeted temperature regardless of the cooling technique, device, or cooling interval. TTM without TFD was defined as the use of a water jet pan, underarm ice pack, or cooling blanket that was not connected to an automated thermostat controlling system. TTM with TFD was defined as the use of specific commercial devices, such as Arctic Sun® (Medivance Corp, Louisville, KY, USA), GAYMAR (Gaymar Industries, Orchard Park, NY, USA), Blanketrol III (Cincinnati Sub-Zero Products, Cincinnati, OH, USA), and EMCOOLS Flex.Pad ${ }^{\mathrm{m}}$ (EMCOOLS, Vienna, Austria) or intravascular devices, such as CoolGard 3000 Thermal Regulation System (Alsius Corporation, Irvine, CA). These devices were connected to an automated feedback thermostat that controlled the temperature of the circulating saline based on the patients' rectal or esophageal temperature.

The study population was divided into four groups according to the targeted temperatures and implementation of TFD: $36^{\circ} \mathrm{C}$ TTM with TFD (36TFD), $33^{\circ} \mathrm{C}$ TTM with TFD (33TFD), $36^{\circ} \mathrm{C}$ TTM without TFD (36N TFD), and $33^{\circ} \mathrm{C}$ TTM without TFD (33NTFD) (Fig. 1). Information on individual factors, including age, sex, underlying disease, laboratory data, initial vital signs, Utstein factors, such as primary electrocardiogram (ECG) (shockable rhythm versus non-shockable rhythm), location, witnessed arrest, and bystander cardiopulmonary resuscitation (CPR) was collected. Data on total arrest time, defined as the time from onset of the arrest until return of spontaneous circulation, and hospital factors, such as the provision of coronary interventions and TTM induction time, were collected.

\section{Outcome Measures}

The main clinical outcome was a favorable neurological status at hospital discharge, which was defined as a cerebral performance category (CPC) score of 1 or 2 .

\section{Statistical Analyses}

As all eligible study participants during the designated study period were included, sample size estimation using power analysis was not performed; instead, a retrospective power analysis was implemented. Continuous variables are presented as means \pm standard deviation and categorical variables are presented as absolute or relative frequencies. Group comparisons were performed using analysis of variance for continuous variables and the chi-squared test for categorical variables. The Bonferroni post hoc test was used to perform pairwise comparisons of groups. Multivariable logistic regression analyses with adjustment for the influence of confounders were performed to determine the association between neurological outcome and TFD or targeted temperatures. To assess whether associations between TFD use and neurological outcome differed by targeted temperatures $\left(33^{\circ} \mathrm{C}\right.$ or $\left.36^{\circ} \mathrm{C}\right)$, the interactions between TFD and targeted temperatures were tested in a multivariable logistic regression model. The $\mathrm{P}$ value for statistical significance was set at $<0.05$ for an interaction term.

Additional multivariable logistic regression analyses were performed to estimate the independent association between neurological outcome and the four groups, which were divided according to the combination of TFD and targeted temperatures, with the 33NFD group as the reference group. Pairwise comparisons (Bonferroni corrected for multiple comparisons) of the adjusted odds ratio were subsequently evaluated to further investigate any differences between the individual groups in more detail. All multivariable logistic regression models were adjusted for age, sex, witness, bystander CPR, primary ECG rhythm, total arrest time, and provision of coronary intervention. P-values $<0.05$ were considered to be statistically significant at the $95 \%$ confidence level. The

$P$ value of the Bonferroni correction for multiple comparisons was set at $0.0083(0.05 / 6)$.

All statistical analyses were conducted using SAS software (version 9.2; SAS Institute Inc., Cary, NC, USA) and R software for Windows (version 3.2.5; the R foundation for statistical computing, Vienna, Austria [http://www.R-project.org/]).

\section{Results}

Enrolment and clinical outcome data for the OHCA patients registered in the KoCARC registry from October 2015 to June 2020 are presented in Fig. 1. Of the patients who received TTM, 91 were excluded due to age < 18 years, provision of extracorporeal CPR, and missing data on body temperature and devices, resulting in 938 patients in the final analysis. Of these patients, 302 (32.2\%) patients were discharged with good neurological outcomes, i.e., CPC score of 1 or 2.

Clinical characteristics of the participants divided into the four groups according to TFD use and targeted temperatures are shown in Table 1. The 33NTFD group exhibited the least favorable neurological compared to the other groups. There were no significant differences at baseline for the demographic and clinical characteristics of the study participants with the exception of the provision of coronary intervention. The provision of coronary intervention was performed more frequently in patients in the 36TFD group than in patients in the 33 TFD or 33NTFD groups $(46.8 \%$ vs. $29.7 \%$; $P<0.001,46.8 \%$ vs. $31.1 \%$; $P=$ 0.007 , respectively) (Table 1 ). 
Table 1. Clinical characteristics of study patients stratified according to targeted temperature and temperature control device 


\begin{tabular}{|c|c|c|c|c|c|c|c|c|c|c|c|}
\hline \multirow[t]{3}{*}{ Variables } & \multirow{3}{*}{$\begin{array}{l}\text { Total } \\
\mathrm{N}=938\end{array}$} & \multicolumn{2}{|c|}{ TTM at $36^{\circ} \mathrm{C}$} & \multicolumn{2}{|c|}{ TTM at $33^{\circ} \mathrm{C}$} & \multirow{3}{*}{$\begin{array}{l}\text { overall } \\
P\end{array}$} & \multicolumn{5}{|c|}{ Post-hoc analysis p-value } \\
\hline & & (1) NTFD & \multirow{2}{*}{$\begin{array}{l}\text { (2) TFD } \\
\mathrm{N}=237\end{array}$} & \multirow{2}{*}{$\begin{array}{l}\text { (3) NTFD } \\
\mathrm{N}=104\end{array}$} & \multirow{2}{*}{$\begin{array}{l}\text { (4) TFD } \\
\mathrm{N}=536\end{array}$} & & \multirow[t]{2}{*}{1 vs 2} & \multirow[t]{2}{*}{1 vs 3} & \multirow{2}{*}{$\begin{array}{l}1 \text { vs } \\
4\end{array}$} & \multirow[t]{2}{*}{2 vs 3} & \multirow[t]{2}{*}{2 vs 4} \\
\hline & & $N=61$ & & & & & & & & & \\
\hline Age (years) & $59.1 \pm 17.1$ & $60.5 \pm 16.8$ & $61.1 \pm 15.8$ & $60.3 \pm 19.4$ & $57.9 \pm 17.2$ & 0.077 & 0.807 & 0.959 & 0.260 & 0.712 & 0.016 \\
\hline $\begin{array}{l}\text { Male sex [n } \\
(\%)]\end{array}$ & $688(73.4)$ & $41(67.2)$ & $175(73.8)$ & $76(73.1)$ & $396(73.9)$ & 0.733 & 0.301 & 0.423 & 0.265 & 0.883 & 0.991 \\
\hline \multicolumn{12}{|l|}{$\begin{array}{l}\text { Vital sign after } \\
\text { ROSC }\end{array}$} \\
\hline $\begin{array}{l}\text { Systolic blood } \\
\text { pressure } \\
(\mathrm{mmHg})\end{array}$ & $130 \pm 76$ & $124 \pm 32$ & $140 \pm 125$ & $129 \pm 50$ & $126 \pm 46$ & 0.272 & 0.231 & 0.725 & 0.850 & 0.360 & 0.058 \\
\hline $\begin{array}{l}\text { Diastolic blood } \\
\text { pressure } \\
(\mathrm{mmHg})\end{array}$ & $76 \pm 27$ & $75.553 \pm 23$ & $78 \pm 27$ & $79 \pm 33$ & $75 \pm 26$ & 0.667 & 0.687 & 0.578 & 0.896 & 0.778 & 0.326 \\
\hline $\begin{array}{l}\text { Heart rate } \\
\text { (bpm) }\end{array}$ & $100 \pm 34$ & $106 \pm 33$ & $100 \pm 32$ & $92 \pm 39$ & $101 \pm 33$ & 0.231 & 0.304 & 0.063 & 0.450 & 0.183 & 0.559 \\
\hline \multicolumn{12}{|l|}{$\begin{array}{l}\text { Medical } \\
\text { history, [n (\%)] }\end{array}$} \\
\hline Hypertension & $203(21.66)$ & $8(13.11)$ & $55(23.21)$ & 21(20.19) & 119(22.24) & 0.362 & 0.085 & 0.249 & 0.099 & 0.538 & 0.768 \\
\hline $\begin{array}{l}\text { Diabetes } \\
\text { mellitus }\end{array}$ & $151(16.12)$ & $9(14.75)$ & $37(15.61)$ & $22(21.15)$ & $83(15.51)$ & 0.527 & 0.869 & 0.310 & 0.876 & 0.213 & 0.972 \\
\hline $\begin{array}{l}\text { Chronic } \\
\text { pulmonary } \\
\text { disease }\end{array}$ & 28(2.99) & $3(4.92)$ & 11(4.64) & $2(1.92)$ & $12(2.24)$ & 0.189 & $>0.999$ & 0.360 & 0.191 & 0.358 & 0.071 \\
\hline $\begin{array}{l}\text { Cardiovascular } \\
\text { disease }\end{array}$ & 156(16.65) & 11(18.03) & $46(19.41)$ & $15(14.42)$ & $84(15.70)$ & 0.551 & 0.807 & 0.539 & 0.637 & 0.269 & 0.204 \\
\hline $\begin{array}{l}\text { Cerebral } \\
\text { vascular } \\
\text { disease }\end{array}$ & $41(4.38)$ & $3(4.92)$ & $12(5.06)$ & $6(5.77)$ & $20(3.74)$ & 0.617 & $>0.999$ & $>.9999$ & 0.721 & 0.788 & 0.394 \\
\hline $\begin{array}{l}\text { Chronic kidney } \\
\text { disease }\end{array}$ & $51(5.44)$ & 1(1.64) & $14(5.91)$ & $6(5.77)$ & $30(5.61)$ & 0.601 & 0.321 & 0.262 & 0.354 & 0.960 & 0.868 \\
\hline Malignancy & $44(4.70)$ & $4(6.56)$ & $14(5.91)$ & $4(3.85)$ & $22(4.11)$ & 0.551 & 0.770 & 0.469 & 0.327 & 0.433 & 0.275 \\
\hline \multicolumn{12}{|l|}{$\begin{array}{l}\text { Cardiac arrest } \\
\text { characteristics }\end{array}$} \\
\hline $\begin{array}{l}\text { Witnessed } \\
\text { collapse, [n } \\
(\%)]\end{array}$ & $706(75.3)$ & 49(81.67) & $173(73.0)$ & 76(73.79) & $408(76.55)$ & 0.201 & 0.158 & 0.251 & 0.371 & 0.219 & 0.475 \\
\hline $\begin{array}{l}\text { Bystander } \\
\text { CPR, [n (\%)] }\end{array}$ & $490(54.14)$ & $33(58.93)$ & $116(49.15)$ & $46(49.46)$ & 295(56.73) & 0.164 & 0.188 & 0.262 & 0.752 & 0.960 & 0.053 \\
\hline $\begin{array}{l}\text { First } \\
\text { monitored } \\
\text { rhythm, [n (\%)] }\end{array}$ & & & & & & 0.416 & 0.738 & 0.484 & 0.608 & 0.179 & 0.783 \\
\hline $\begin{array}{l}\text { Non- } \\
\text { shockable }\end{array}$ & $517(56.32)$ & $35(58.33)$ & 132(55.93) & $62(63.92)$ & $288(54.86)$ & & & & & & \\
\hline Shockable & $401(43.68)$ & $25(41.67)$ & $104(44.07)$ & $35(36.08)$ & $237(45.14)$ & & & & & & \\
\hline $\begin{array}{l}\text { Cardiac arrest } \\
\text { in public } \\
\text { location } \\
\text { [n (\%)] }\end{array}$ & $424(45.20)$ & $26(42.62)$ & $107(45.15)$ & $37(35.58)$ & 254(47.39) & 0.235 & 0.872 & 0.666 & 0.649 & 0.237 & 0.197 \\
\hline $\begin{array}{l}\text { No-flow time } \\
\text { (min) }\end{array}$ & $6.8 \pm 6.7$ & $6.2 \pm 7.0$ & $6.5 \pm 6.4$ & $6.7 \pm 8.1$ & $7.0 \pm 6.6$ & 0.713 & 0.802 & 0.696 & 0.415 & 0.819 & 0.342 \\
\hline $\begin{array}{l}\text { Low-flow time } \\
\text { (min) }\end{array}$ & $25.6 \pm 19.3$ & $24.4 \pm 18.6$ & $26.6 \pm 19.9$ & $26.4 \pm 21.0$ & $25.1 \pm 18.8$ & 0.710 & 0.447 & 0.532 & 0.813 & 0.945 & 0.323 \\
\hline $\begin{array}{l}\text { Duration from } \\
\text { arrest to ROSC } \\
\text { (min) }\end{array}$ & $32.3 \pm 20.3$ & $30.7 \pm 20.5$ & $33.0 \pm 20.5$ & $33.1 \pm 21.8$ & $32.0 \pm 20.0$ & 0.819 & 0.421 & 0.469 & 0.621 & 0.992 & 0.532 \\
\hline $\begin{array}{l}\text { Coronary } \\
\text { reperfusion } \\
\text { intervention, [n } \\
\text { (\%)] }\end{array}$ & $322(34.48)$ & 23(37.70) & $111(46.84)$ & $32(31.07)$ & $156(29.27)$ & $<0.001^{*}$ & 0.201 & 0.384 & 0.174 & $0.007^{\star \star}$ & $<0.001^{* *}$ \\
\hline
\end{tabular}




\begin{tabular}{|c|c|c|c|c|c|c|c|c|c|c|c|}
\hline $\begin{array}{l}\text { Induction time } \\
\text { of TTM (hour) }\end{array}$ & $4.19 \pm 3.7$ & $3.8 \pm 2.2$ & $4.5 \pm 5.3$ & $4.0 \pm 2.7$ & $4.1 \pm 2.5$ & 0.784 & 0.422 & 0.832 & 0.686 & 0.530 & 0.429 \\
\hline \multicolumn{12}{|l|}{$\begin{array}{l}\text { Laboratory } \\
\text { data after } \\
\text { ROSC }\end{array}$} \\
\hline $\begin{array}{l}\text { Hemoglobin } \\
\text { (mg/dL) }\end{array}$ & $12.7 \pm 2.7$ & $12.9 \pm 2.4$ & $12.5 \pm 2.9$ & $13.2 \pm 2.4$ & $12.8 \pm 2.6$ & 0.189 & 0.240 & 0.609 & 0.611 & 0.038 & 0.216 \\
\hline $\begin{array}{l}\text { Platelets } \\
\left(10^{3} / \mathrm{uL}\right)\end{array}$ & $205 \pm 83$ & $220 \pm 114$ & $204 \pm 77$ & $203 \pm 74$ & $205 \pm 83$ & 0.602 & 0.193 & 0.241 & 0.208 & 0.972 & 0.843 \\
\hline PT(INR) & $1.45 \pm 1.69$ & $1.46 \pm 1.55$ & $1.45 \pm 1.20$ & $1.34 \pm 0.38$ & $1.47 \pm 2.02$ & 0.956 & 0.984 & 0.714 & 0.958 & 0.649 & 0.901 \\
\hline $\begin{array}{l}\text { Sodium } \\
(\mathrm{mEq} / \mathrm{L})\end{array}$ & $139.5 \pm 24.7$ & $138.0 \pm 5.3$ & $139.5 \pm 6.2$ & $139.2 \pm 5.7$ & $139.8 \pm 33.0$ & 0.963 & 0.678 & 0.766 & 0.603 & 0.928 & 0.892 \\
\hline $\begin{array}{l}\text { Potassium } \\
\text { (mEq/L) }\end{array}$ & $5.4 \pm 12.8$ & $4.3 \pm 1.0$ & $4.9 \pm 3.2$ & $8.3 \pm 32.4$ & $5.3 \pm 8.7$ & 0.154 & 0.775 & 0.069 & 0.598 & 0.036 & 0.707 \\
\hline $\begin{array}{l}\text { Chloride } \\
(\mathrm{mEq} / \mathrm{L})\end{array}$ & $101.9 \pm 11.1$ & $101.5 \pm 6.3$ & $103.0 \pm 13.2$ & $102.4 \pm 7.3$ & $101.3 \pm 11.1$ & 0.294 & 0.379 & 0.659 & 0.854 & 0.656 & 0.062 \\
\hline Base excess & $10.0 \pm 12.8$ & $5.9 \pm 14.6$ & $9.9 \pm 10.1$ & $11.6 \pm 11.0$ & $10.0 \pm 13.8$ & 0.367 & 0.196 & 0.076 & 0.147 & 0.427 & 0.922 \\
\hline $\mathrm{PH}$ & $7.07 \pm 0.22$ & $7.09 \pm 0.21$ & $7.08 \pm 0.20$ & $7.03 \pm 0.19$ & $7.06 \pm 0.24$ & 0.250 & 0.631 & 0.085 & 0.331 & 0.077 & 0.426 \\
\hline $\begin{array}{l}\text { Arterial lactate } \\
(\mathrm{mmol} / \mathrm{L})\end{array}$ & $10.50 \pm 5.04$ & $10.67 \pm 4.51$ & $11.02 \pm 4.32$ & $10.86 \pm 7.14$ & $10.18 \pm 5.02$ & 0.186 & 0.647 & 0.835 & 0.499 & 0.811 & 0.038 \\
\hline BUN (mg/dL) & $25.7 \pm 26.4$ & $19.8 \pm 10.2$ & $25.1 \pm 17.6$ & $27.3 \pm 32.5$ & $26.4 \pm 29.9$ & 0.318 & 0.179 & 0.099 & 0.078 & 0.527 & 0.567 \\
\hline $\begin{array}{l}\text { Creatinine } \\
(\mathrm{mg} / \mathrm{dL})\end{array}$ & $2.4 \pm 7.3$ & $1.4 \pm 1.2$ & $3.3 \pm 12.2$ & $1.9 \pm 2.0$ & $2.1 \pm 3.3$ & 0.221 & 0.127 & 0.736 & 0.541 & 0.239 & 0.075 \\
\hline AST (IU/mL) & $289 \pm 753$ & $168 \pm 132$ & $293 \pm 863$ & $283 \pm 471$ & $304 \pm 781$ & 0.686 & 0.288 & 0.397 & 0.225 & 0.923 & 0.869 \\
\hline ALT (IU/mL) & $203 \pm 594$ & $127 \pm 138$ & $226 \pm 779$ & $164 \pm 264$ & $208 \pm 564$ & 0.684 & 0.287 & 0.730 & 0.362 & 0.438 & 0.720 \\
\hline $\begin{array}{l}\text { Bilirubin } \\
(\mathrm{mg} / \mathrm{dL})\end{array}$ & $0.68 \pm 1.29$ & $0.53 \pm 0.39$ & $0.75 \pm 2.19$ & $0.55 \pm 0.46$ & $0.68 \pm 0.75$ & 0.548 & 0.264 & 0.916 & 0.405 & 0.246 & 0.560 \\
\hline $\begin{array}{l}\text { Albumin } \\
\text { (g/dL) }\end{array}$ & $3.68 \pm 7.29$ & $3.42 \pm 0.67$ & $4.40 \pm 14.18$ & $3.41 \pm 0.67$ & $3.42 \pm 0.67$ & 0.471 & 0.396 & 0.996 & 0.996 & 0.306 & 0.129 \\
\hline $\begin{array}{l}\text { Glucose } \\
(\mathrm{mg} / \mathrm{L})\end{array}$ & $285 \pm 134$ & $294 \pm 148$ & $282 \pm 119$ & $307 \pm 128$ & $281 \pm 141$ & 0.392 & 0.564 & 0.564 & 0.515 & 0.145 & 0.944 \\
\hline $\begin{array}{l}\text { Total } \\
\text { cholesterol } \\
(\mathrm{mg} / \mathrm{dL})\end{array}$ & $142 \pm 66$ & $147 \pm 38$ & $149 \pm 50$ & $161 \pm 152$ & $136 \pm 49$ & 0.060 & 0.890 & 0.372 & 0.431 & 0.290 & 0.095 \\
\hline $\begin{array}{l}\text { Favorable } \\
\text { neurological } \\
\text { outcome [n } \\
(\%)]\end{array}$ & $302(32.20)$ & $20(32.79)$ & $84(35.44)$ & 11(10.58) & 187(34.89) & $<0.001^{\star}$ & 0.698 & $<0.001^{\star \star}$ & 0.744 & $<0.001^{\text {** }}$ & 0.882 \\
\hline
\end{tabular}

${ }^{*} \mathrm{P}<0.05,{ }^{*} \mathrm{P}<0.0083$ ( $\mathrm{P}$ value threshold after a Bonferroni correction for 6 comparisons)

Variables are expressed as mean \pm SD for continuous variables or number (\%) for categorical variables.

Abbreviations: TTM, targeted temperature management; NTFD, TTM without temperature feedback devices; TFD, TTM with temperature feedback devices; ROSC, return of spontaneous circulation; CPR, cardiopulmonary resuscitation; PT(INR), Prothrombin time (international normalized ratio); BUN, blood urea nitrogen; AST, aspartate aminotransferase; ALT, alanine aminotransferase.

No significant differences were noted in the clinical characteristics between the TFD and NTFD groups. Further, the proportion of favorable neurological outcomes was higher in the TFD groups $(35.1 \%$ vs $18.8 \%, \mathrm{P}<0.01)$ (Supplementary Table S1). The TFD groups were independently associated with more favorable neurological outcomes (odds ratio [OR]: $2.37,95 \% \mathrm{Cl}: 1.39-4.05, \mathrm{P}=0.002$ ) than the NTFD groups (Table 2). TFD use and targeted temperatures were seen to have a significant association with the neurological outcomes $(P=0.032)$ of OHCA patients with TTM via the interaction term between the two factors, after adjusting for age, sex, witness, bystander CPR, primary ECG rhythm, total arrest time, and provision of coronary intervention (Table 2). 
Table 2

Multivariable logistic regression analysis of favorable neurological outcome for TFD use and targeted temperatures.

\begin{tabular}{|c|c|c|c|c|c|}
\hline \multirow[t]{2}{*}{ Variable } & \multicolumn{4}{|c|}{ Favorable neurological outcome } & \multirow[t]{2}{*}{$P$ for interaction } \\
\hline & AOR $(95 \% \mathrm{Cl})$ & $\mathbf{P}$ & AOR $(95 \% \mathrm{Cl})$ & $\mathbf{P}$ & \\
\hline Age & $0.967(0.956-0.978)$ & $<0.001^{\star}$ & $0.966(0.955-0.977)$ & $<0.001^{\star}$ & \\
\hline Male sex & $0.944(0.615-1.451)$ & 0.794 & $0.913(0.595-1.401)$ & 0.676 & \\
\hline Duration from arrest to ROSC duration (per $1 \mathrm{~min}$ ) & $0.946(0.935-0.958)$ & $<0.001^{*}$ & $0.947(0.936-0.958)$ & $<0.001^{*}$ & \\
\hline Witnessed collapse & $1.831(1.176-2.852)$ & $0.007 *$ & $1.833(1.178-2.853)$ & $0.007 *$ & \\
\hline Bystander CPR & $1.202(0.829-1.742)$ & 0.332 & $1.222(0.844-1.768)$ & 0.289 & \\
\hline Initial shockable rhythm & $6.070(4.134-8.914)$ & $<0.001^{*}$ & $6.213(4.234-9.118)$ & $<0.001^{*}$ & \\
\hline Provision coronary intervention & $2.320(1.588-3.389)$ & $<0.001^{*}$ & $2.136(1.457-3.130)$ & $<0.001^{*}$ & \\
\hline TTM without TFD & \multicolumn{4}{|l|}{ Reference } & \multirow[t]{4}{*}{$0.032^{*}$} \\
\hline TTM with TFD & $2.372(1.390-4.048)$ & $0.002^{*}$ & & & \\
\hline TTM at $36^{\circ} \mathrm{C}$ & & & \multicolumn{2}{|l|}{ Reference } & \\
\hline TTM at $33^{\circ} \mathrm{C}$ & & & $0.706(0.476-1.046)$ & 0.083 & \\
\hline$\star P<0.05$ & & & & & \\
\hline
\end{tabular}

Among the four groups, patients with 33NTFD were independently associated with the least favorable neurological and survival outcomes after adjusting for confounders including age, sex, cause of arrest, witness, bystander CPR, primary ECG rhythm, total arrest time, and provision of coronary interventions (Table 3 and Fig. 2). Patients with 36NTFD exhibited a significantly higher OR for favorable neurological status (OR: 4.28, 95\% Cl: 1.50-12.16) compared with 33NTFD patients after adjusting for covariates (Table 3A). Moreover, the 36NTFD group showed no significant differences in the neurological outcomes at hospital discharge compared with 36TFD or 33TFD groups after adjusting for covariates (Table 3B).

Table 3. Multivariable logistic regression for favorable neurological outcomes (A) and AOR comparisons of the four groups (B).

(A)

\begin{tabular}{|lll|}
\hline Variable & \multicolumn{2}{c|}{ Favorable neurological outcome } \\
\cline { 2 - 3 } & AOR $(95 \% \mathrm{Cl})$ & $\boldsymbol{P}$ \\
\hline Age & $0.967(0.956-0.978)$ & $<0.001^{*}$ \\
\hline Male sex & $0.926(0.601-1.429)$ & 0.729 \\
\hline Duration from arrest to ROSC & $0.947(0.936-0.958)$ & $<0.001^{*}$ \\
\hline Witnessed collapse & $1.836(1.175-2.869)$ & $0.008^{*}$ \\
\hline Bystander CPR & $1.207(0.828-1.758)$ & 0.327 \\
\hline Initial shockable rhythm & $6.284(4.258-9.272)$ & $<0.001^{*}$ \\
\hline Provision coronary intervention & $2.169(1.470-3.201)$ & $<0.001^{*}$ \\
\hline 33 NTFD & Reference & \\
\hline 36 NTFD & $4.275(1.503-12.157)$ & $0.006^{*}$ \\
\hline 33 TFD & $4.334(1.972-9.525)$ & $<0.001^{*}$ \\
\hline 36 TFD & $5.379(2.337-12.381)$ & $<0.001^{*}$ \\
\hline
\end{tabular}

(B) 


\begin{tabular}{|c|c|c|c|c|c|c|c|c|c|}
\hline \multirow[t]{2}{*}{ Variables } & \multicolumn{9}{|c|}{ Favorable neurological outcomes } \\
\hline & $\begin{array}{l}\text { AOR } \\
(95 \% \mathrm{Cl})\end{array}$ & $\begin{array}{l}P \\
\text { 33NTFD }\end{array}$ & vs & $\begin{array}{l}P \\
\text { 36NTFD }\end{array}$ & vs & $\begin{array}{l}P \\
\text { 33TFD }\end{array}$ & vs & $\begin{array}{l}P \\
\text { 36TFD }\end{array}$ & vs \\
\hline 33 NTFD & Reference & Reference & & $0.006 * \star$ & & $<0.001^{\star \star}$ & & $<0.001^{\star \star}$ & \\
\hline 36 NTFD & $\begin{array}{l}4.275(1.503- \\
12.157)\end{array}$ & $0.006^{\star *}$ & & Reference & & 0.972 & & 0.577 & \\
\hline 33 TFD & $\begin{array}{l}4.334(1.972- \\
9.525)\end{array}$ & $<0.001^{\star \star}$ & & 0.972 & & Reference & & 0.328 & \\
\hline 36 TFD & $\begin{array}{l}5.379(2.337- \\
12.381)\end{array}$ & $<0.001^{\star \star}$ & & 0.577 & & 0.328 & & Reference & \\
\hline
\end{tabular}

*P<0.05; $* * \mathrm{P}<0.0083$ ( $\mathrm{P}$ value threshold after a Bonferroni correction for 6 comparisons)

Abbreviations: ROSC, return of spontaneous circulation; CPR, cardiopulmonary resuscitation; $33 \mathrm{NTFD}, 36^{\circ} \mathrm{C}$ TTM with TFD; $36 \mathrm{NTFD}, 33^{\circ} \mathrm{C}$ TTM with TFD; $33 T F D, 36^{\circ} \mathrm{C}$ TTM without TFD; $36 \mathrm{TFD}, 33^{\circ} \mathrm{C}$ TTM without TFD; AOR, adjusted odds ratio (adjusted for age, sex, witnessed, bystander CPR, primary electrocardiogram rhythm, total arrest time, and provision of coronary intervention); $\mathrm{Cl}$, confidence interval.

Finally, in a power analysis based on the results from the present study, we had a power of $96.5 \%$ to detect a difference of neurological outcome among the four groups.

\section{Discussion}

The primary purpose of this study was to investigate the optimal target temperature in TTM without TFD in terms of neurological outcomes in post cardiac arrest patients. The main finding of this study was that TTM with 33NTFD was significantly associated with a poorer neurological outcome than TTM with TFD and even 36NTFD. Despite differences in cost, medical resource use, and invasiveness, there were no significant differences in patient outcomes among the 36TFD, 33TFD, and 36NTFD groups in our cohort. Our findings suggest that a targeted temperature of $36^{\circ} \mathrm{C}$ rather than $33^{\circ} \mathrm{C}$ is associated with more favorable neurological outcomes if TFD is unavailable. Moreover, our results implied that 36NTFD could provide a therapeutic effectiveness non-inferior to TFD in OHCA patients treated with TTM. To the best of our knowledge, this study is the first to identify the optimal targeted temperature in TTM without TFD with regards to neurological outcomes in OHCA patients.

Consistent with the findings of previous research, no significant differences were observed in the clinical outcomes between the targeted temperatures of $33^{\circ} \mathrm{C}$ and $36^{\circ} \mathrm{C}$ regardless of the use of TTM devices. In this study, neurological outcomes were more favorable in the TFD groups than in the NTFD groups (34.7\% vs $16.7 \%, p<0.001)$. Substantial evidence corroborates the superior effectiveness of temperature feedback devices in achieving, maintaining, and rewarming targeted temperatures compared to the absence of such devices [1,9-11]. A recent systematic review reported that the use of temperature feedback systems in TTM was associated with a higher probability of favorable neurological outcomes compared to NFD [9]. The concept of "high-quality TTM" has been proposed as a method to increase the effectiveness of TTM. The use of a high-quality TTM system equipped with automatic temperature feedback systems is recommended to manage targeted temperatures with minimal fluctuations [1]. If body temperatures are not adequately monitored and adjusted, the beneficial effects of TTM may be compromised due to delays in TTM induction, unintentional overcooling or hyperthermia, or spontaneous and rapid rewarming [1, 20].

Nevertheless, issues of cost and availability of TFD in real-world settings should be considered. In this study, $16.7 \%$ of post-cardiac arrest patients received TTM without temperature feedback devices. Commercial surface cooling or intravascular temperature management equipment and automated thermostat systems are often costly and not fully covered by health insurance programs [8]. Moreover, the high density of post-cardiac arrest patients in a few advanced hospitals results in the shortage of TTM equipment, and the demand for technologically sophisticated devices for TTM often exceeds their availability [10, 12]. Advanced devices for TTM may not be readily available in clinical settings in developing countries [21]. Therefore, physicians often encounter clinical situations in which TFD is unavailable. TTM without TFD is cheaper and more readily available compared to TTM with TFD, but the accuracy of temperature control is poor and may lead to unfavorable clinical outcomes [1,22]. Despite the significant association between NTFD and poor clinical outcomes, it remains unclear whether the association differs according to targeted temperature $\left(33^{\circ} \mathrm{C}\right.$ vs. $\left.36^{\circ} \mathrm{C}\right)$.

The current study demonstrated that TTM at $33^{\circ} \mathrm{C}$ was significantly associated with clinical deterioration compared to TTM at $36^{\circ} \mathrm{C}$ when $\mathrm{TFD}$ was not used. Adverse side effects have been associated with $\mathrm{TTM}$ at $33^{\circ} \mathrm{C}$. Casamento et al. reported similar adherence rates with temperature guidelines between $33^{\circ} \mathrm{C}$ and $36^{\circ} \mathrm{C}$ [15], but the occurrence of arrhythmias and cardiovascular dysfunction was higher in patients with TTM at $33^{\circ} \mathrm{C}$. Düggelin et al. reported that TTM at $36^{\circ} \mathrm{C}$ was associated with higher adherence to precise temperatures and a lower rate of adverse effects, such as bradycardia [16]. Merchant et al. reported that maintaining accurate temperature control with conventional cooling methods (without TFD) in TTM at $33^{\circ} \mathrm{C}$ was difficult and was associated with unintentional overcooling episodes [22]. Further, they demonstrated that TTM at $33^{\circ} \mathrm{C}$ using ice packs and conventional cooling blankets led to poorer clinical outcomes and lowered the threshold for adverse events. The authors suggested that accurate induction and maintenance in TTM at $33^{\circ} \mathrm{C}$ was dependent on the selected cooling method and appropriate adjustments in response to ongoing temperature fluctuations [22]. Generally, $\mathrm{TTM}$ at $33^{\circ} \mathrm{C}$ is more strongly associated with unintentional overcooling and adverse events, such as cardiac dysfunction and arrhythmias, whereas $\mathrm{TTM}^{\mathrm{T}}$ at $36^{\circ} \mathrm{C}$ is more strongly associated with a delayed start of TTM and hyperthermic episodes [13-16]. Given that the body's temperature control mechanisms for temperature maintenance operate optimally at $36^{\circ} \mathrm{C}, \mathrm{TTM}$ at $36^{\circ} \mathrm{C}$ may be more likely to induce rebound hyperthermia $[13,23]$. Although the precise mechanisms 
underscoring poorer clinical outcomes in 33NTFD than in 36NTFD remain unclear, several factors, such as unintentional overcooling, hyperthermia, and a delayed start of TTM, may contribute, as they are associated with poor clinical outcomes [1]. Hyperthermic episodes and delayed induction may be prevented by adequate administration of neuromuscular blocking agents and implementation of standard TTM strategies [1, 6]. Most participating hospitals in our study have implemented treatment protocols for patients receiving TTM according to international guidelines. No differences were observed in induction time between TTM at $33^{\circ} \mathrm{C}$ and $36^{\circ} \mathrm{C}$ in this study. Considerable research has attempted to identify the optimal approaches to prevent shivering and subsequent hyperthermia during TTM, and reliable protocols and guidelines have been published in this regard [6, 24-28]. There have been significant improvements in treatment strategies and physicians' attention to minimize delays in induction, shivering, and hyperthermia based on evidence, such as the American Heart Association guidelines in the practice of resuscitation [28]. These advances may have contributed to improved prognosis in the 36NTFD group. In contrast, appropriate guidelines to prevent overcooling in TTM using non-feedback devices, such as ice bags and cooling blankets, are less common than those for preventing hyperthermic events [22]. There were no significant differences in patient outcomes among the 36TFD, 33TFD, and 36NTFD groups in our study. A recent large, prospective, randomized study in OHCA patients demonstrated that targeted hypothermia at $33^{\circ} \mathrm{C}$ did not show significant benefit in clinical outcomes compared to targeted normothermia with early treatment of fever [29]. The combined results of our study and this recent trial imply that 36NTFD with active prevention of hyperthermia could achieve meaningful clinical improvement in OHCA patients with TTM as compared with 33NTFD.

There are several limitations to this study. First, the assignment of TTM devices and targeted temperatures could not be randomly allocated due to the observational nature of the study. Although we adjusted for potential confounders in the multivariable logistic regression model, unmeasured bias may have affected device selection and targeted temperatures. Second, in our study setting, the selection of TTM devices and targeted temperatures was determined according to the physicians' preference and family consent to pay the TTM fees. As such, the patient's socioeconomic and cultural background may have been sources of bias in the study. Third, the KoCARC registry did not collect data on adverse events and body temperature during TTM. Therefore, we were unable to evaluate the prevalence of adverse events, unintentional overcooling, and hyperthermic episodes in each treatment group. Finally, there is an alternative explanation for the current results is clinician bias in selecting the TTM strategy. Several studies have reported that treating physicians are more likely to select TTM at $33^{\circ} \mathrm{C}$ for patients they believe to have more severe hypoxic insults in order to maximize the protective effects of TTM against brain damage $[1,30]$. If a clinical judgment was made based on patient severity, the allocation of more severe patients to the $\operatorname{TTM}_{\text {group }}$ at $33^{\circ} \mathrm{C}$ would bias our results to favor 36NTFD. However, there were no significant differences in baseline clinical characteristics between the individual treatment groups and the logistic regression analysis was performed adjusting for severity and prognostic confounding factors. Nevertheless, signs of cerebral injury, such as malignant status myoclonus of patients, were not collected in the KoCARC registry, and the relatively small sample size of the 36NTFD group limits the generalizability of these results. Further well-designed trials are needed to clarify the clinical differences between the 36NTFD and TFD groups.

\section{Conclusions}

In OHCA patients receiving TTM without automated devices using a temperature feedback system, the adjusted predicted probability of favorable neurological outcomes and survival at discharge was greater at $36^{\circ} \mathrm{C}$ than at $33^{\circ} \mathrm{C}$. Moreover, the 36 NTFD group displayed similar therapeutic effects as the TFD groups. This suggests that targeted temperature management at $36^{\circ} \mathrm{C}$ shows therapeutic effectiveness if automated devices using a temperature feedback system is unavailable.

\section{Abbreviations}

Targeted temperature management (TTM), out-of-hospital cardiac arrest (OHCA), temperature management devices with feedback systems (TFDs), temperature management without temperature feedback devices (NTFD), $36^{\circ} \mathrm{C}$ TTM with TFD (36TFD), $33^{\circ} \mathrm{C}$ TTM with TFD (33TFD), $36^{\circ} \mathrm{C}$ TTM without TFD (36NTFD), $33^{\circ} \mathrm{C}$ TTM without TFD (33NTFD), odds ratios (ORs), confidence interval (Cl), emergency departments (EDs), Korean Cardiac Arrest Research Consortium (KoCARC), emergency medical services (EMSs), cerebral performance category (CPC), cardiopulmonary resuscitation (CPR), electrocardiogram (ECG).

\section{Declarations}

\section{Ethics approval and consent to participate}

This study was approved by the institutional review boards of each participating institute, and informed consent was waived due to the observational nature of the study. The study has therefore been performed in accordance with the ethical standards laid down in the 1964 Declaration of Helsinki and its later amendments.

\section{Consent for publication}

All authors have read and approved the submission of the manuscript.

\section{Availability of data and materials}

Data are available on reasonable request. Data are available on request.

\section{Competing interests}

All authors declare no conflicts of interest.

\section{Funding}


This study was supported by a faculty research grant from Yonsei University College of Medicine for 2018 (6-2018-0064 to TK) and a grant from the National Research Foundation of Korea (NRF) grant funded by the Ministry of Science, ICT \& Future Planning (NRF- 2018R1C1B6006159 to JSY). The funding bodies played no role in the design of the study and collection, analysis, and interpretation of data and in writing the manuscript.

\section{Authors' contributions}

TK, JSY, and SPC conceived and planned the study and were mainly responsible for its design. TK, and JSY collected data. TK, JSY, HSL, SJ, and SPC were mainly responsible for analyzing the data. TK and JSY wrote the first draft of the manuscript. All authors (TK, JSY, HSL, SJ, YSP, and SPC) contributed to the interpretation of the findings and reviewed the manuscript. HSL, SJ, YSP, and SPC reviewed the statistical analyses and made changes to the content of the manuscript. All authors (TK, JSY, HSL, SJ, YSP, and SPC) also provided intellectual contribution to the manuscript.

\section{Acknowledgments}

We would like to acknowledge and thank to investigators from all participating hospitals of KoCARC: Woon Yong Kwon (Seoul National University Hospital), Sang Kuk Han, Phil Cho Choi (Kangbuk Samsung Medical Center), Sang O Park, Jong Won Kim (Konkuk University Medical Center), Han Sung Choi, Jong Seok Lee (Kyung Hee University Hospital), Sung Hyuk Choi, Young Hoon Yoon (Korea University Guro Hospital), Su Jin Kim, Kap Su Han (Korea University Anam Hospital), Min Seob Sim, Gun Tak Lee (Samsung Medical Center), Shin Ahn (Asan Medical Center), Jong Whan Shin, Hui Jai Lee (SMG-SNU Boramae Medical Center), Keun Hong Park, Hahn Bom Kim (Seoul Medical Center), In Cheol Park, Yoo Seok Park (Yonsei University Severance Hospital), Tae Young Kong (Yonsei University Gangnam Severance Hospital), Chu Hyun Kim (Inje University Seoul Paik Hospital), Youngsuk Cho (Hallym University Kangdong Sacred Heart Hospital), Gu Hyun Kang, Yong Soo Jang (Hallym University Kangnam Sacred Heart Hospital), Tai Ho Im, Jae Hoon Oh (Hanyang University Seoul Hospital), Seok Ran Yeom, Sung Wook Park (Pusan National University Hospital), Jae Hoon Lee (Dong-A University Hospital), Jae Yun Ahn (Kyungpook National University Hospital), Kyung Woo Lee, Tae Chang Jang (Daegu Catholic University Medical Center), Jae-hyug Woo (Gachon University Gil Medical Center), Woon Jeong Lee, Seon Hee Woo (The Catholic University of Korea Incheon St. Mary's Hospital), Sung Hyun Yun, Jin Won Park (Catholic Kwandong University International St. Mary's Hospital), Sun Pyo Kim, Yong Jin Park (Chosun University Hospital), Jin Woong Lee, Wonjoon Jeong (Chungnam National University Hospital), Sung Soo Park, Jae Kwang Lee (Konyang University Hospital), Ryeok Ahn, Wook Jin Choi (Ulsan University Hospital), Young Gi Min, Eun Jung Park (Ajou University Hospital), You Hwan Jo, Joong Hee Kim (Seoul National University Bundang Hospital), In Byung Kim, Ki Ok Ahn (Myongji Hospital), Han Jin Cho (Korea University Ansan Hospital), Seung Cheol Lee, Sang Hun Lee (Dongguk University Ilsan Hospital), Young Sik Kim (Bundang Jesaeng Hospital), Jin Sik Park (Sejong Hospital), Dai Han Wi (Wonkwang University Sanbon Hospital), Ok Jun Kim, Min Woo Kang (Cha University Bundang Medical Center), Sang Ook Ha, Won Seok Yang (Hallym University Pyeongchon Sacred Heart Hospital), Soon Joo Wang, Hang A Park (Hallym University Dongtan Sacred Heart Hospital), Jun Hwi Cho, Chan Woo Park (Kangwon National University Hospital), An Mu Eob, Tae Hun Lee (Hallym University Chuncheon Sacred Heart Hospital), Sang Chul Kim, Hoon Kim (Chungbuk National University Hospital), Han Joo Choi , Chan Young Koh (Dankook University Hospital), Hyung Jun Moon, Dong Kil Jeong (Soonchunhyang University Cheonan Hospital), Tae Oh Jung, Jae Chol Yoon (Chonbuk National University Hospital), Seung Yong Lee, Young Tae Park (Dongguk University Gyeongju Hospital), Jin Hee Jeong, Soo Hoon Lee (Gyeongsang National University Hospital), Ji Ho Ryu, Mun Ki Min (Pusan National University Yangsan Hospital ), Won Kim, Yi Sang Moon (Cheju Halla General Hospital), Sung Wook Song, Woo Jung Kim (Jeju National University Hospital), Joon-myoung Kwon, Eui Hyuk Kang (Mediplex Sejong Hospital), Sang Chan Jin, Tae-kwon Kim (Keimyung University Dongsan Medical Center), Hyuk Joong Choi (Hanyang University Guri Hospital), Seong Chun Kim (Gyeongsang National University Changwon Hospital), In Soo Cho (Hanil General Hospital).

To steering committee, comprised of following individuals: Sung Oh Hwang (Chair, Wonju Severance Christian Hospital), Sang Do Shin (Chair of Steering Committee, Seoul National University hospital), Hyuk Jun Yang (Advisory Committee, Gachon University Gil hospital), Sung Phil Chung (Data Safety and Management Board, Yonsei University Gangnam Severance Hospital), Sung Woo Lee (Security and Monitoring Board, Korea University Anam hospital), Kyung Jun Song (Secretariat, SMG-SNU Boramae Medical Center), Seung Sik Hwang (Epidemiology and Prevention Research Committee, Seoul National University), Gyu Chong Cho (Community Resuscitation Research Committee, Hallym University Kangdong Sacred Heart Hospital), Hyun Wook Ryoo (Emergency Medical Service Resuscitation Research Committee, Kyungpook National University Hospital), Kyoung Chul Cha (Hospital Resuscitation Research Committee, Wonju Severance Christian Hospital), Won Young Kim (Hypothermia and Post-Resuscitation Care Research Committee, Asan Medical Center), Sang Hoon Na (Cardiac Care Resuscitation Research Committee, Seoul National University Hospital), Young Ho Kwack (Pediatric Resuscitation Research Committee, Seoul National University hospital)

To member of Secretariat: Jeong Ho Park (Seoul National University Hospital), Sun Young Lee (Seoul National University Hospital), and Sung Kyung Kim (Seoul National University Hospital).

To National Fire Agency for providing prehospital EMS data. And to Korean Association of Cardiopulmonary Resuscitation (KACPR) for support.

\section{References}

1. Taccone FS, Picetti E, Vincent JL. High Quality Targeted Temperature Management (TTM) After Cardiac Arrest. Crit Care. 2020;24:6.

2. Cronberg T, Lilja G, Horn J, Kjaergaard J, Wise MP, Pellis T, Hovdenes J, Gasche Y, Åneman A, Stammet P, Erlinge D, Friberg H, Hassager C, Kuiper M, Wanscher M, Bosch F, Cranshaw J, Kleger GR, Persson S, Undén J, Walden A, Winkel P, Wetterslev J, Nielsen N. Neurologic Function and Health-Related Quality of Life in Patients Following Targeted Temperature Management at $33^{\circ} \mathrm{C}$ vs $36^{\circ} \mathrm{C}$ After Out-of-Hospital Cardiac Arrest: A Randomized Clinical Trial. JAMA Neurol. 2015;72:634-41.

3. Mild therapeutic hypothermia. to improve the neurologic outcome after cardiac arrest. N Engl J Med. 2002;346:549-56. 
4. Bernard SA, Gray TW, Buist MD, Jones BM, Silvester W, Gutteridge G, Smith K. Treatment of comatose survivors of out-of-hospital cardiac arrest with induced hypothermia. N Engl J Med. 2002;346:557-63.

5. Nielsen N, Wetterslev J, Cronberg T, Erlinge D, Gasche Y, Hassager C, Horn J, Hovdenes J, Kjaergaard J, Kuiper M, Pellis T, Stammet P, Wanscher M, Wise MP, Åneman A, Al-Subaie N, Boesgaard S, Bro-Jeppesen J, Brunetti I, Bugge JF, Hingston CD, Juffermans NP, Koopmans M, Køber L, Langørgen J, Lilja G, Møller JE, Rundgren M, Rylander C, Smid O, Werer C, Winkel P, Friberg H. Targeted temperature management at $33^{\circ} \mathrm{C}$ versus $36^{\circ} \mathrm{C}$ after cardiac arrest. $\mathrm{N}$ Engl J Med. 2013;369:2197-206.

6. Panchal AR, Bartos JA, Cabañas JG, Donnino MW, Drennan IR, Hirsch KG, Kudenchuk PJ, Kurz MC, Lavonas EJ, Morley PT, O'Neil BJ, Peberdy MA, Rittenberger JC, Rodriguez AJ, Sawyer KN, Berg KM. Part 3: Adult Basic and Advanced Life Support: 2020 American Heart Association Guidelines for Cardiopulmonary Resuscitation and Emergency Cardiovascular Care. Circulation. 2020;142:366-s468.

7. Donnino MW, Andersen LW, Berg KM, Reynolds JC, Nolan JP, Morley PT, Lang E, Cocchi MN, Xanthos T, Callaway CW, Soar J. Temperature Management After Cardiac Arrest: An Advisory Statement by the Advanced Life Support Task Force of the International Liaison Committee on Resuscitation and the American Heart Association Emergency Cardiovascular Care Committee and the Council on Cardiopulmonary, Critical Care, Perioperative and Resuscitation. Resuscitation. 2016;98:97-104.

8. Kim KH, Shin SD, Song KJ, Ro YS, Kim YJ, Hong KJ, Jeong J, Park JH, Kim TH, Kong SY. Cooling methods of targeted temperature management and neurological recovery after out-of-hospital cardiac arrest: A nationwide multicenter multi-level analysis. Resuscitation. 2018;125:56-65.

9. Calabró L, Bougouin W, Cariou A, De Fazio C, Skrifvars M, Soreide E, Creteur J, Kirkegaard H, Legriel S, Lascarrou JB, Megarbane B, Deye N, Taccone FS. Effect of different methods of cooling for targeted temperature management on outcome after cardiac arrest: a systematic review and meta-analysis. Crit Care. 2019;23:285.

10. Hoedemaekers CW, Ezzahti M, Gerritsen A, van der Hoeven JG. Comparison of cooling methods to induce and maintain normo- and hypothermia in intensive care unit patients: a prospective intervention study. Crit Care. 2007;11:R91.

11. Vaity C, Al-Subaie N, Cecconi M. Cooling techniques for targeted temperature management post-cardiac arrest. Crit Care. 2015;19:103.

12. Storm C, Nee J, Sunde K, Holzer M, Hubner P, Taccone FS, Friberg H, Lopez-de-Sa E, Cariou A, Schefold JC, Ristagno G, Noc M, Donker DW, Andres J, Krawczyk P, Skrifvars MB, Penketh J, Krannich A, Fries M. A survey on general and temperature management of post cardiac arrest patients in large teaching and university hospitals in 14 European countries-The SPAME trial results. Resuscitation. 2017;116:84-90.

13. Johnson NJ, Danielson KR, Counts CR, Ruark K, Scruggs S, Hough CL, Maynard C, Sayre MR, Carlbom DJ. Targeted Temperature Management at 33 Versus 36 Degrees: A Retrospective Cohort Study. Crit Care Med. 2020;48:362-9.

14. Bray JE, Stub D, Bloom JE, Segan L, Mitra B, Smith K, Finn J, Bernard S. Changing target temperature from $33^{\circ} \mathrm{C}$ to $36^{\circ} \mathrm{C}$ in the ICU management of out-ofhospital cardiac arrest: A before and after study. Resuscitation. 2017;113:39-43.

15. Casamento A, Minson A, Radford S, Mårtensson J, Ridgeon E, Young P, Bellomo R. A comparison of therapeutic hypothermia and strict therapeutic normothermia after cardiac arrest. Resuscitation. 2016;106:83-8.

16. Düggelin R, Maggiorini M, Voigtsberger S, Schwarz U, Hilty MP. Increased protocol adherence and safety during controlled normothermia as compared to hypothermia after cardiac arrest. J Crit Care. 2020. https://doi.org/10.1016/j.jcrc.2020.09.019.

17. Yoon JC, Kim YJ, Ahn S, Jin YH, Lee SW, Song KJ, Shin SD, Hwang SO, Kim WY. Factors for modifying the termination of resuscitation rule in out-ofhospital cardiac arrest. Am Heart J. 2019;213:73-80.

18. Kim JY, Hwang SO, Shin SD, Yang HJ, Chung SP, Lee SW, Song KJ, Hwang SS, Cho GC, Moon SW, Kim K, Kim WY, Oh S, Kwak YH. Korean Cardiac Arrest Research Consortium (KoCARC): rationale, development, and implementation. Clin Exp Emerg Med. 2018;5:165-76.

19. Kong T, Chung SP, Lee HS, Kim S, Lee J, Hwang SO, Shin SD, Song KJ, Cha KC, You JS. The Prognostic Usefulness of The Lactate/Albumin Ratio For Predicting Clinical Outcomes In Out-Of-Hospital Cardiac Arrest: A Prospective, Multicentre Observational Study (KoCARC Study). Shock. 2019. https://doi.org/10.1097/shk.0000000000001405.

20. Kim JG, Ahn C, Shin H, Kim W, Lim TH, Jang BH, Cho Y, Choi KS, Lee J, Na MK. Efficacy of the cooling method for targeted temperature management in post-cardiac arrest patients: A systematic review and meta-analysis. Resuscitation. 2020;148:14-24.

21. Suraseranivongse S, Chawaruechai T, Saengsung P, Komoltri C. Outcome of cardiopulmonary resuscitation in a 2300-bed hospital in a developing country. Resuscitation. 2006;71:188-93.

22. Merchant RM, Abella BS, Peberdy MA, Soar J, Ong ME, Schmidt GA, Becker LB, Vanden Hoek TL. Therapeutic hypothermia after cardiac arrest: unintentional overcooling is common using ice packs and conventional cooling blankets. Crit Care Med. 2006;34:490-4.

23. Tan CL, Knight ZA. Regulation of Body Temperature by the Nervous System. Neuron. 2018;98:31-48.

24. Logan A, Sangkachand P, Funk M. Optimal management of shivering during therapeutic hypothermia after cardiac arrest. Crit Care Nurse. 2011;31:e1830.

25. Honasoge A, Parker B, Wesselhoff K, Lyons N, Kulstad E. First Use of a New Device for Administration of Buspirone and Acetaminophen to Suppress Shivering During Therapeutic Hypothermia. Ther Hypothermia Temp Manag. 2016;6:48-51.

26. Paul M, Bougouin W, Dumas F, Geri G, Champigneulle B, Guillemet L, Ben Hadj Salem O, Legriel S, Chiche JD, Charpentier J, Mira JP, Sandroni C, Cariou A. Comparison of two sedation regimens during targeted temperature management after cardiac arrest. Resuscitation. 2018;128:204-10.

27. Picetti E, Oddo M, Prisco L, Helbok R, Taccone FS. A Survey on Fever Monitoring and Management in Patients With Acute Brain Injury: The SUMMA Study. J Neurosurg Anesthesiol. 2019;31:399-405.

28. Callaway CW, Donnino MW, Fink EL, Geocadin RG, Golan E, Kern KB, Leary M, Meurer WJ, Peberdy MA, Thompson TM, Zimmerman JL. Part 8: PostCardiac Arrest Care: 2015 American Heart Association Guidelines Update for Cardiopulmonary Resuscitation and Emergency Cardiovascular Care.

Page $11 / 13$ 
Circulation. 2015;132:465-82.

29. Dankiewicz J, Cronberg T, Lilja G, Jakobsen JC, Levin H, Ullén S, Rylander C, Wise MP, Oddo M, Cariou A, Bělohlávek J, Hovdenes J, Saxena M, Kirkegaard H, Young PJ, Pelosi P, Storm C, Taccone FS, Joannidis M, Callaway C, Eastwood GM, Morgan MPG, Nordberg P, Erlinge D, Nichol AD, Chew MS, Hollenberg J, Thomas M, Bewley J, Sweet K, Grejs AM, Christensen S, Haenggi M, Levis A, Lundin A, Düring J, Schmidbauer S, Keeble TR, Karamasis GV, Schrag C, Faessler E, Smid O, Otáhal M, Maggiorini M, Wendel Garcia PD, Jaubert P, Cole JM, Solar M, Borgquist O, Leithner C, Abed-Maillard S, Navarra L, Annborn M, Undén J, Brunetti I, Awad A, McGuigan P, Bjørkholt Olsen R, Cassina T, Vignon P, Langeland H, Lange T, Friberg H, Nielsen N. Hypothermia versus Normothermia after Out-of-Hospital Cardiac Arrest. N Engl J Med. 2021;384:2283-94.

30. Du L, Ge B, Ma Q, Yang J, Chen F, Mi Y, Zhu H, Wang C, Li Y, Zhang H, Yang R, Guan J, Zhang Y, Jin G, Zhu H, Xiong Y, Wang G, Zhu Z, Zhang H, Zhang Y, Zhu J, Li J, Lan C, Xiong H. Changes in cardiac arrest patients' temperature management after the publication of 2015 AHA guidelines for resuscitation in China. Sci Rep. 2017;7:16087.

\section{Supplementary}

Appendix A. Supplementary data

Supplementary information accompanies this paper at https://

\section{Figures}

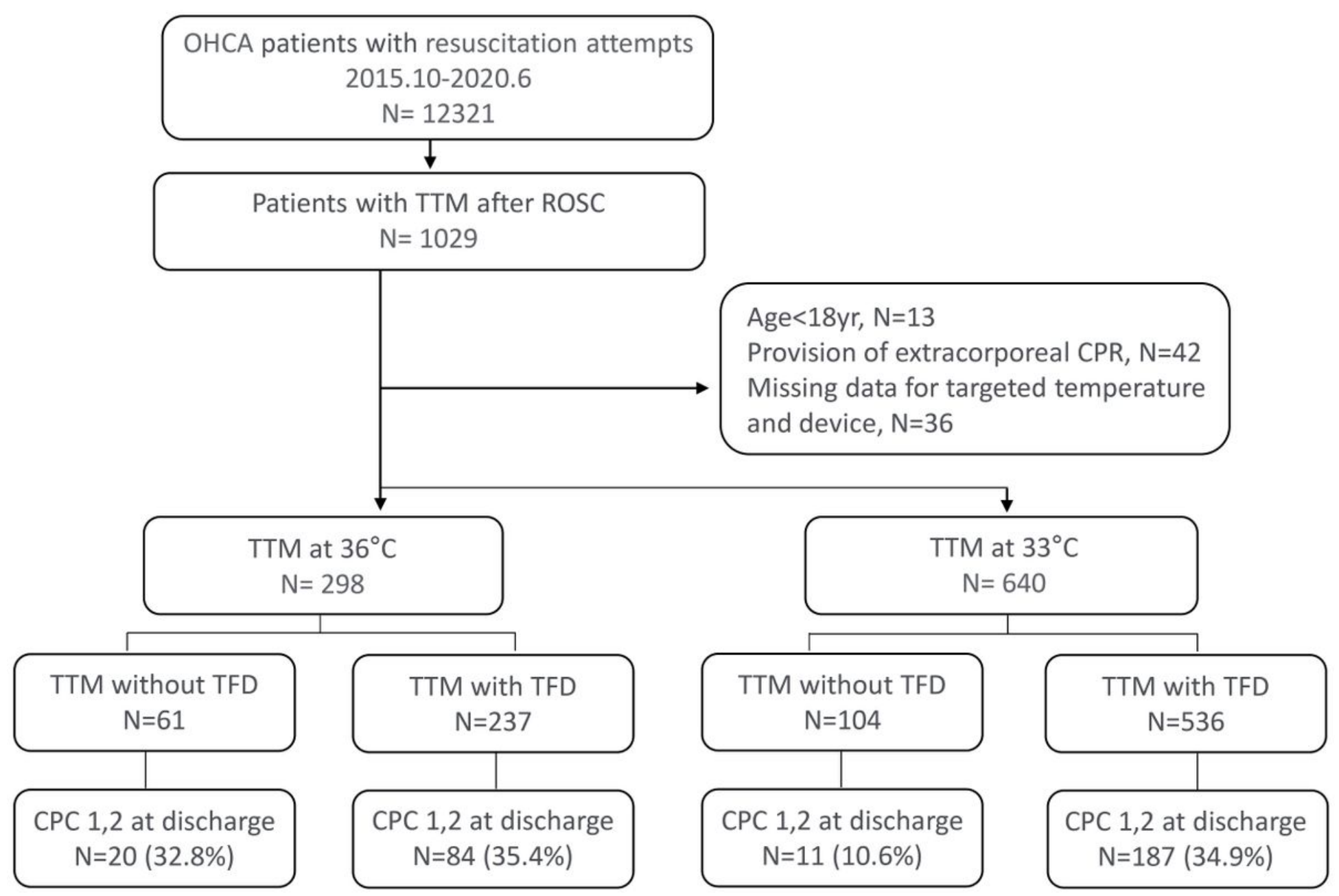

Figure 1

Flow diagram of the study population. TTM, targeted temperature management; ROSC, return of spontaneous circulation; TFD, temperature management devices with feedback systems; CPC, cerebral performance category; OHCA, out-of-hospital cardiac arrest; CPR, cardiopulmonary resuscitation 


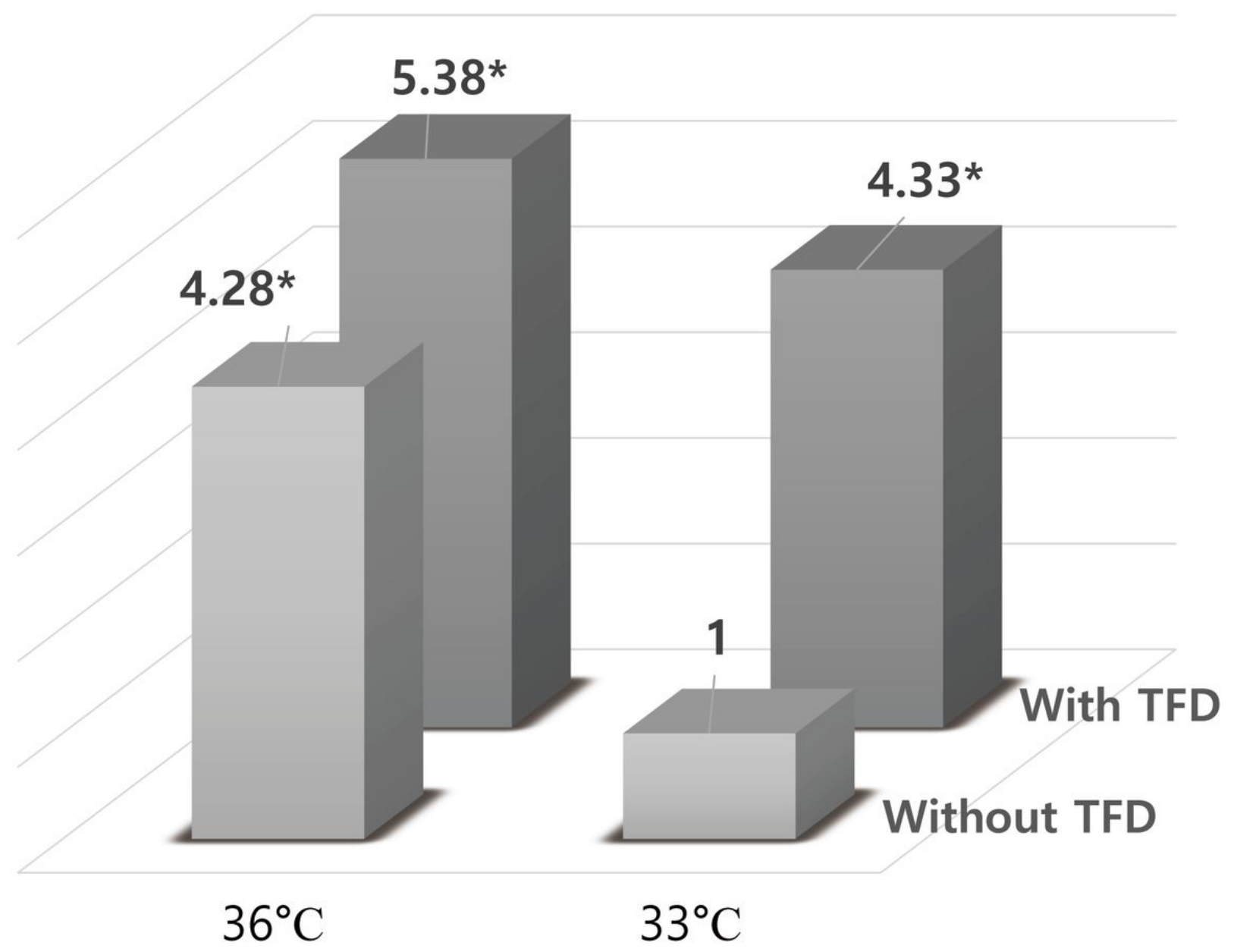

Figure 2

Adjusted odds ratio for favorable neurological status according to targeted temperature and temperature feedback device. TFD, temperature management devices with feedback systems

\section{Supplementary Files}

This is a list of supplementary files associated with this preprint. Click to download.

- Tables1.docx 